\title{
Diabetes-Associated Kidney and Vascular Complications: Mechanisms of Disease Progression and Alternative Therapeutic Options
}

Sara Gallo, Maddalena Gili, Gabriele Togliatto and Maria Felice Brizzi

Department of Medical Sciences, University of Torino, Italy

"Corresponding author: Maria Felice Brizzi, Department of Medical Sciences, University of Torino, Corso Dogliotti 14, 10126, Torino, Italy, E-mail: mariafelice.brizzi@unito.it

Received date: January 14, 2013; Accepted date: February 25, 2014; Published date: March 3, 2014

Copyright: $@ 2014$ Gallo S, et al. This is an open-access article distributed under the terms of the Creative Commons Attribution License, which permits unrestricted use, distribution, and reproduction in any medium, provided the original author and source are credited.

\begin{abstract}
Vascular complications in diabetes are an emergent health care problem. Accelerated endothelial dysfunction in pathological settings connoted by hyperlipidaemia and hyperglycaemia is a crucial step for the development and the progression of atherosclerosis. Previous data support the central role of Advanced Glycated End-products (AGEs) and oxidation or glycation of Low Dense Lipoproteins (LDLs) in the impaired vascular remodelling associated with diabetes. Hyperglycemia, via NADPH Oxidase (NOX) enzymatic activity, upholds the production of Reactive Oxygen Species (ROS), which in turn mediate tissue damage and long-lasting "metabolic memory". Nonetheless, in diabetic setting, ROS act as secondary messenger to strictly control stemness of visceral-derived adipose stem cells and to promote transcriptional and post-transcriptional events, also involving small non-coding microRNAs (miRs). In this article we provide an overview on the events elicited by acute and chronic hyperglycemia that account for vascular and kidney diseases. The deleterious effects of LDL and fatty acids on endothelial progenitor cells in condition connoted by hyperglycemia are also discussed. Moreover, as current therapeutic approaches failed to improve endothelial dysfunction/disease progression and consequently long-term outcomes in diabetics with vascular complications, particular attention has been devoted to describe efforts made to identify novel therapeutic options, for the management of one of the most relevant health care problems world wide. Finally, as targeting of epigenetic mechanisms is a future challenge, relevant data supporting their deep involvement in long-lasting "metabolic memory" have been also addressed.
\end{abstract}

Keywords: Diabetes; Vascular complications; Kidney disease; ROS; Epigenetics; miRNAs; UnAG

\section{Introduction}

Cardiovascular diseases are the major causes of morbidity and mortality in patients with type 2 diabetes [1]. Several lines of evidences indicate that the accelerated impairment of endothelial functions and the loss of an efficient blood vessel perfusion are crucial for the development of atherosclerosis in diabetes [2,3]. It is well established that atherosclerosis is a process, which results from interaction among plasma lipoproteins, cellular components (monocyte/macrophages, $\mathrm{T}$ lymphocytes, endothelial cells and smooth muscle cells, endothelial progenitor cells) and extracellular matrix [4].

Modified low density lipoproteins (particularly the oxidized small dense LDL (sdLDL) act as crucial players in the initiation of the proinflammatory reaction which leads to vessel injury [4]. Besides abnormal LDL, the advanced glycated end-products (AGEs), resulting from long-term diabetes, are crucial determinants of diabetic vascular complications. Generation of oxidative stress and dysfunctional mitochondria, the lack of efficient antioxidant machinery, as well as the overproduction of growth factors and cytokines are mainly involved in disease progression [5-7]. In this review, we will focus on the molecular mechanisms leading to vascular damage and kidney disease associated with diabetes. Particular attention will be paid to the deleterious effects of LDL or protein oxidation or glycation and their downstream signals. The last part of the review will be focused on the mechanisms accounting for the long-lasting "metabolic memory" as well as on novel therapeutic approaches targeting oxidative stress.

\section{Vascular Damage}

Low Density Lipoproteins (LDL) and endothelial cells (ECs)

The concept of LDL oxidative modification was first built up by Steinberg et al. in 1989 [8]. From then to now a number of preclinical and clinical studies have extensively documented that qualitative (glycation and/or oxidation) and quantitative LDL abnormalities in diabetes exert a pivotal role in the development and progression of atherosclerosis [9]. Modified LDL, no longer efficiently bound by the canonical LDL receptor, acquire the ability to bind a number of scavenger receptors expressed by endothelial cells (EC) and macrophages, such as LOX-1 (oxLDL lecitin like Receptor 1), CD36, SR-PSOX [10] and the receptor for AGE (RAGE) [11]. LDL binding to these non canonical receptors brings on a vast array of proatherogenic responses intimately linked to lesion progression in diabetic setting [12-14]. Of interest oxLDL are much more vulnerable to glycoxidation [15] and, as suggested by the use of RAGE-deficient murine aortic ECs, for oxLDL-mediated proatherogenic signals RAGE is crucial [16]. This implies that ROS generation elicited by binding of "AGEs" to RAGE is central in the activation of the redox-sensitive nuclear transcription factor $\mathrm{kB}(\mathrm{NF}-\mathrm{kB})$ and the transcription of a variety of atherosclerosis-related genes, including PAI-1, tissue factor, VCAM-1, ICAM-1, MCP-1, VEGF, and RAGE itself [17-19]. Alternatively we found that small and dense LDL recovered from diabetic patients (dm-LDL), via RAGE, induce ROS generation, activate the signal transducers and activators of transcription (STAT)5B and inhibit EC cell-cycle progression [12,13]. The observation that such deleterious cue was mediated by the STAT5B 
transcription activity on the cyclin dependent kinase inhibitor p21waf $[12,13]$ delineates a new mechanism through which RAGE can stimulate intracellular events to drive changes in gene expression.

Essential to diabetes is the development of chronic hyperglycemia resulting in AGE formation [19]. As stated above this process also involves LDL which, by undergoing AGE-modification, acquire more relevant pro-atherogenic properties and accelerate atherosclerosis. As the result of such additional qualitative abnormality, LDL switch on a cascade of intracellular events, that besides STAT5 and PKC [20,21], also include the activation of the Erk1/Erk2 MAP and Src kinases $[13,22]$. More recent reports have highlighted novel mechanisms in RAGE signaling. In particular Shui et al. [23] have shown that the activation of the mTOR pathway, known to be associated with cardiac hypertrophy and atherosclerosis, is also crucial for LOX-1 expression in response to AGE-mediated signals. More interestingly, they provide evidences for a novel protective effect of metformin administration that open new insight to moving forward to human studies. Indeed, metformin by interfering with the mTOR pathway was found to reduce LOX-1 expression on ECs [23].

Accumulating evidences indicate that gly-LDL by oxidative stress generation can also induce thrombogenic reactions. At this regard, Sangle et al. [24] provide evidences that gly-LDL lead to heat shock factor 1 (HSF 1)-mediated PAI 1 production. By using both in vitro and in vivo models they elegantly showed that NOX, and H-Ras/Raf-1 signaling pathways are implicated in the up-regulation of HSF1 or PAI-1 in ECs [24].

Over the last few years a number of studies have demonstrated that $\mathrm{T}$ and $\mathrm{B}$ lymphocytes and dendritic cells express RAGE $[25,26]$, suggestinga role of RAGE in adaptive immune responses as well. Although this novel RAGE role is still debated [25,27,28], it has been suggested that ligand-mediated RAGE activation could be relevant for effective $\mathrm{T}$ lymphocytepriming during acute inflammatory responses. Based on the notion that long-term diabetes recapitulates chronic diseases it is conceivable to hypothesize that, in diabetes, RAGE signaling could maintain a sustained inflammation andtissue damage also by acting on immune cells [19].

An immunological response to gly and oxLDL has been also reported and seems to predict, more efficiently than traditional risk factors, vascular disease progression in diabetes [29,30]. In keeping with this observation it has been reported that the progression of diabetic retinopathy positively correlates with the level of immunecomplexes containing gly- and ox-LDL [31]. As gracefully shown by the authors immune-complex binding to macrophages leads to oxidative stress, mitochondrial dysfunction and retinal pericytes apoptosis [31]. More recently, Bernal Lopez et al. [32] also demonstrated a positive correlation between the levels of anti-ox-LDL IgM and the expression of genes involved in inflammation, apoptosis, plaque disruption, lipidic metabolism and cellular turnover. However, whether this correlation simply reflects plaque instability or the inflammatory milieu of diabetic patients is still open to debate [32].

Apart from glycation and oxidation, in diabetic patients LDL can get into homocysteinisation [33]. Owing to lipoproteinglycation, LDL appear to be more susceptible to bind homocysteine derivatives, and a direct correlation between homocystamide LDL derivates and the $\mathrm{HbAlc}$ levels has been reported. Thus, additional EC oxidative damage can be induced by homocystamide LDL-mediated peroxynitrite production [33].
One last word must be dedicated to the work of Pirillo et al. [34] which demonstrated how modified HDL (through the 15 lypoxigenasis) lose their protective effect and become pro atherogenic (Figure 1).

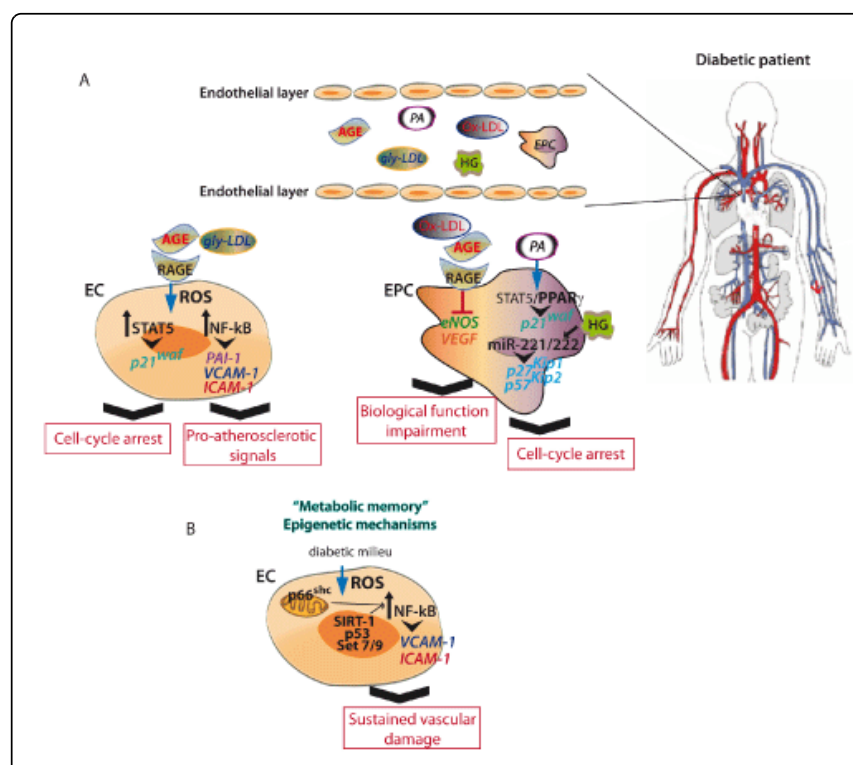

Figure 1: Schematic representation of mechanisms involved in diabetes-associated vascular damage (A) Circulating factors involved in diabetes-associated vascular damage are schematically described.Advanced glycation end-products (AGE), glycated LDL (gly-LDL), oxidized LDL (Ox-LDL), high glucose (HG) palmitic acid (PA) are crucial determinants of vascular damage. When AGE/ gly-LDL interact with the receptors for AGE (RAGE) on EC, they stimulate generation of reactive oxygen species (ROS). This results in STAT5 activation and p21waf expression, leading to cell-cycle arrest, or in the activation of the nuclear factor $\mathrm{kB}$ (NF-kB). NF-kB in turn transcriptionally regulates its target genes: plasminogen activator inhibitor-1 (PAI-1)/vascular cell adhesion molecule-1 (VCAM-1)/intercellular adhesion molecule-1 (ICAM-1). In diabetic condition circulating endothelial progenitor cells (EPC) are reduced in number and impaired in function. AGE and Ox-LDL via RAGE down-regulate VEGF and eNOS activity. Moreover, while high palmitic acid (PA) concentrations activate STAT5/ PPAR $\gamma$ complex leading to $\mathrm{p} 21$ waf expression, HG up-regulates miR-221/222 expression to control p21waf, p27Kip1 and p57Kip2 content that results in EPC cell-cycle arrest. (B) Persistent hyperglycaemia induces epigenetic changes that determine the so called "metabolic memory". The relevant mediators of epigenetic changes that sustain vascular damage are depicted: mitochondrial p66shc, sirtuin-1 (SIRT-1), p53, and Set7/9.

\section{Hyperlipemia/hyperglycaemia and endothelial progenitor cell dysfunctions}

The bone marrow pool of endothelial progenitor cells (EPCs) has attracted particular attention due to their potential clinical application $[6,35]$. However, EPCs are reduced in number in patients with type 1 or type 2 diabetes [36-38]. Moreover, their functional capabilities are impaired in these pathological conditions [36-38]. Of note, in diabetic patients with peripheral vascular complications a further and 
progressive impairment of EPC number has been reported [37]. A number of studies indicate that besides hyperglycaemia different metabolic stress factors associated with diabetes contribute to such quantitative and qualitative abnormalities [39,40]. Among these, oxidized LDL (ox-LDL), alterated fatty acids and AGE are included $[36-38,41]$.

AGE, by binding to RAGE, induce apoptosis and impair EPC migration and tube-like structure formation by affecting VEGFsignalling pathway and eNOS activity [42]. Liang et al. [43] reported that such dysfunctional EPC phenotype could be reverted by administration of anti-AGE antibodies or rosiglitazone, a well-known PPARgamma agonist $[43,44]$. That the inflammatory milieu, via the up-regulation of RAGE, is also crucial for AGE-mediated effects is supported by experiments performed on EPC exposed to C-reactive protein [45].

Along with ox-LDL, alteration of lipid and in particular of fatty acid (NEFA) metabolism is common in type 2 diabetes [46]. Indeed, metabolic profiling of plasma NEFAs in type 2 diabetes patients has discovered different biomarkers, including Palmitic Acid (PA) [47]. $\mathrm{PA}$ is a ligand for cell surface receptors and for transcription factor receptors, the PPARs. PPARs are crucial regulators of genes involved in lipid and glucose metabolism, vascular functions, and inflammation [48]. We have recently demonstrated that EPCs recovered from diabetic patients, displaying high concentrations of PA, or EPCs cultured in diabetic concentration of PA are unable to undergo cellcycle progression [49]. We found that, as expected, diabetic concentrations of PA induce the expression of PPAR gamma. However, in this particular condition PPAR gamma binds to the promoter region of STAT5 and negatively influences its transcription. Despite the reduction of STAT5 content, the STAT5/PPAR gamma complex is still formed, but changes its target. It binds to the p21 $1^{\text {waf }}$ promoter and induces its transcription. So that EPCs undergo cellcycle arrest.

In addition to precise transcriptional regulation, vascular remodeling requires post-transcriptional regulation, involving small non-coding microRNAs (miRs) [50]. miRs are highly conserved, noncoding small RNAs which regulate gene expression at the posttranscriptional level [51]. Recently, a miRNA signature in insulin target tissues and a plasma miRNA profile in type 2 diabetes have been reported [52]. Different families of miRs, including miR-221/222, have been shown to be involved in the control of endothelial cell fate and vascular remodeling [53]. In particular, miR-221/222-driven posttranscriptional regulation of cyclin-dependent kinase (Cdk) inhibitors (CKIs) p2 $7^{\text {Kip } 1}$ and $\mathrm{p} 57^{\mathrm{Kip} 2}$ have been involved in mechanical vascular diseases [54]. Consistently, we demonstrated that acute or chronic exposure to high glucose concentrations prevented cell-cycle progression of both ECs and EPCs by modulating the expression of p27 Kip1 and p57 Kip2 [55]. Moreover, we found that high-glucose and AGEs were also able to inhibit vessel formation in an in vivo model of angiogenesis by regulating the expression of miR221/222. Along with miR-221/222, miR-320 [56] and miR-503 [57] have been shown to contribute to the impaired vascular remodeling in preclinical models of diabetes. In particular, Caporali et al. [57] demonstrated that forced expression of mirR-503 in ECs leads to inhibition of vascular growth in diabetic mice subjected to hind limb ischemia. This effect relies on miR-503-driven post-transcriptional regulation of the cell-cycle related CCNE1 and cdc25A genes [57]. The potential clinical implication of miR-503 in diabetes-associated vascular complications is sustained by the observation that diabetic patients display increased plasma miR-503 levels [57]. Thus, these data, besides confirming that deregulation of miR-221/222, miR-320 and miR-503 expression could be involved in high glucose-driven anti-angiogenic signals, identify miRNAs as potential targets for pharmacological intervention to improve vascular dysfunction in conditions connoted by altered glucose metabolism [55-57].

\section{Kidney Disease}

\section{AGE effects on mesangial cells}

Diabetic nephropathy consists of an early onset of glomerular and tubular hypertrophy and a late extracellular matrix accumulation leading to a progressive expansion of the mesangial cells (MCs) [58]. Mesangial cell hypertrophy mainly depends on intracellular signals elicited by transforming growth factor beta (TGF- $\beta$ ) [59]. Cell-cycle independent or dependent mechanisms are implicated in the control of cell hypertrophy [60]. TGF $\beta$-induced cell-cycle dependent hypertrophy involves the synthesis of structural protein such as $\mathrm{p} 21^{\text {waf }}$ and $\mathrm{p} 27^{\mathrm{Kip} 1}$ key regulators of G1 phase progression (TGF $\beta$ ) [61]. At this regard, we demonstrated that AGE, but not high glucose (HG), were able to increase p2 $1^{\text {waf }}$ while inhibit cyclin D1 expression in MCs [62]. We also showed that, in response to both TGF- $\beta$ and AGE, cell cycle arrest of MCs is the result of STAT5 binding to the promoter region of $\mathrm{p} 21^{\text {waf }}[62]$. As stated above, along with MC hypertrophy, extracellular matrix accumulation contributes to kidney disease in diabetic setting. We provided evidences that while STAT5 controls TGF- $\beta$ - and AGE-mediated MC hypertrophy it was not involved in collagen production. Moreover, the increase of immunoreactivity for the activated STAT5 and p21 $1^{\text {waf }}$ in kidney biopsies from early to advanced stage of diabetic nephropathy sustains the possibility that these molecular events could also be crucial for human disease [62].

The CXC chemochine ligand 16 (CXCL16) is a transmembrane molecule acting as an adhesion molecule for immune cells, smooth muscle cells and ECs or as a scavenger receptor for ox-LDL in different cell types [63-65]. CXCL16 consists of a surface-bound and a soluble form released by two disintegrin like metalloproteinases ADAM10 and ADAM17 [66,67]. Both CXCL16 and ADAMs are constitutively expressed in human podocytes where CXCL16 is crucial for ox-LDL uptake [68]. Proinflammatory stimuli such as interferon-gamma and tumor necrosis factor-alpha are known to induce the expression of CXCL16 and the release of the soluble form from human podocytes [68]. In addition it has been also reported that the soluble form of CXCL16 contribute to the recruitment of CXCR6-expressing immune cells to sites of active inflammation [69-71]. The crucial role of podocytes in kidney diseases along with the observation that soluble CXCL16 promotes pro-atherogenic signals and can predict long-term mortality in acute coronary syndrome [72] prompted Zaho et al. [73] to evaluate the serum levels of CXCL16 in diabetic patients with or without kidney disease. The results of this study clearly demonstrate that CXCL16 serum levels are higher in diabetic patients with nephropathy than in healthy subjects or diabetic patients without kidney disease. Although the authors concluded that serum CXCL16 levels might represent an indicator of renal injury in diabetic subjects, prospective studies with larger sample sizes are required to assess whether, indeed, CXCL16 could be usefully exploited as a diagnostic biomarker to identify the onset and/or the development of kidney disease in diabetic patients [73]. 


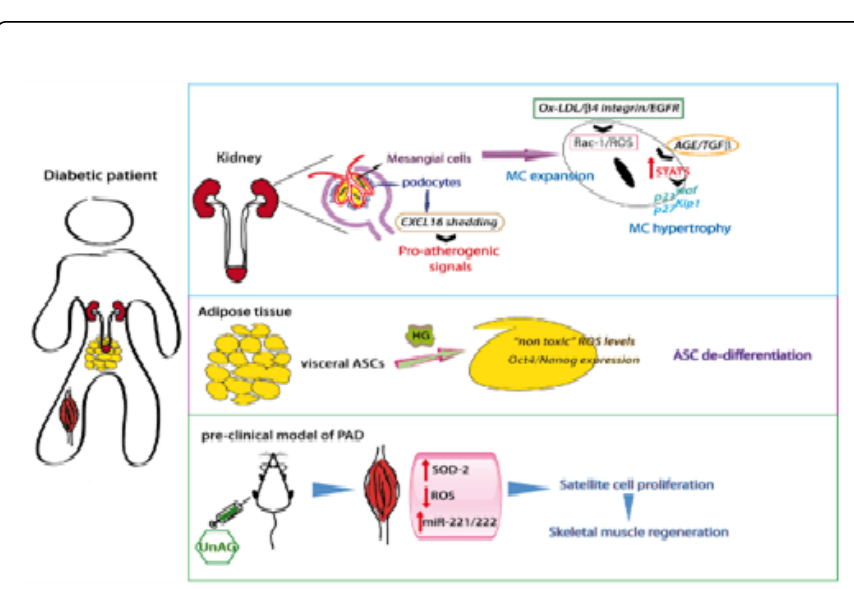

Figure 2: The mechanisms involved in kidney disease and some of the novel therapeutic strategies targeting ROS-mediated damage are schematically represented. Upper panel: The relevant signaling pathways involved in AGE/transforming growth factor beta (TGFß)/Ox-LDL-induced mesangial cell (MC) hypertrophy are depicted. STAT5, Rac-1-mediated ROS generation and the CXC chemokine ligand 16 (CXCL-16) shedding are reported. Middle panel: visceral adipose tissue-derived stem cells (ASCs) in presence of HG de-differentiate into self-renewing cells. Oct-4: octamerbinding transcription factor 4; Nanog: homeobox protein Nanog. Lower panel: schematic representation of the anti-oxidant properties of UnAG in a pre-clinical model of PAD. UnAG administration rescues oxidative-stress-mediated skeletal muscle damage inducing satellite cell proliferation and muscle regeneration. SOD2: Superoxide dismutase 2.

Current therapies for diabetic nephropathy are based on drugs that act on the renin-angiotensin converting enzyme used to control systemic and intraglomerular hypertension [74]. Although a good pressure control reduces the incidence of complications, mortality and progression of nephropathy [75] the achievement of therapeutic goals in diabetic patients is still a major unmet need. Likewise, alternative strategies based on anti-TGF- $\beta$ antibody poorly control the progression of diabetic nephropathy [76]. This suggests that additional therapeutic strategies are still required to preserve diabetic individuals from the end stage renal failure. The involvement of STAT5 in MC hypertrophy and the availability of STAT5 indirect inhibitor(s) [77] raise the possibility that a combinatory approach that targets both TGF- $\beta$ and STAT5 might be exploited in the future to treat such disabling disease.

\section{ox-LDL and MC expansion}

Lipid abnormalities are known to be crucial in the progression from early glomerular injury to progressive glomerulosclerosis [78]. Unlike TGF- $\beta$ and AGE, lipid abnormalities and oxidative stress stimulate MC proliferation and contribute to the development of diabetesassociated renal disease by expanding MCs. However, the molecular mechanisms involved in this process are still undefined. We demonstrate that when cultured in presence of ox-LDL MCs proliferate by activating Akt and Erk1/2 MAPK pathways [79]. Moreover, we first demonstrated that this event is strictly controlled by ROS production and Rac-1 GTPase activation resulting in transactivation of the epidermal growth factor receptor (EGFR). In both physiological and pathological conditions proliferation is strictly controlled by extracellular matrix receptors, the integrins, and the cytokine/tyrosine kinase receptors [80-82]. We provide evidences that MCs challenged with ox-LDL joint $\beta 4$ integrin/EGFR signaling to drive proliferative signals. Therefore the results of this study identify a novel molecular mechanism induced by ox-LDL that in the early stage of renal disease could account for MC expansion via $\beta 4$ integrin activation. By microarray technology Kim et al. [83] provide an alternative mechanism involved in MC hypertrophy upon gly-LDL challenge. Actually, they showed that gly-LDL by activating the Axl/ growth arrest gene 6 axis induce TGF- $\beta$ and its deleterious effects on glomerular MCs [83]. Overall these studies open new perspectives for future pharmacological approaches.

\section{Epigenetic mechanisms and cardiovascular outcomes in diabetic patients}

The signalling pathways involved in glucose-induced vascular damage in diabetes have been deeply characterized [19]. However, a successful therapeutic approach to improve the cardiovascular outcomes in patients with diabetes is still far from being achieved. That hyperglycaemia per se can contribute to diabetes-associated vascular complications has been recently reconsidered in the light of the results of clinical trials indicating that while in new onset diabetes glucose-lowering treatments preserve patients from vascular complications in long-term diabetes the intensive glycaemic control is not effective [84-86]. These observations led to the conclusion that glycaemic environment could be "better or worse remembered" by the vascular system and could contribute to the natural history of diabeticassociated vascular complications [84-86]. The persistence of glucosemediated oxidative stress, AGE production and the positive feedback loop between AGEs and RAGE signals [19], despite a return to good metabolic control, provides the mechanistic justification of the so called "metabolic memory". The "metabolic memory" mainly reflects epigenetic changes driven by hyperglycemia-mediated mitochondrial ROS production [87-91]. Epigenetics depend on histone modifying enzymes, DNA methylation, and chromatin remodeling proteins [89]. These mechanisms are reviewed and consisting in small changes in the epigenome over time [87-91], seem to be also crucial determinants of the early "hyperglycemia memory" sustained by the DCCT/EDIC studies $[84,85]$. Moreover, it has been suggested that epigenetics and in particular DNA methylation, could also explain the awful "metabolic memory" of post-prandial hyperglycemia episodes [92]. Current knowledge supports single mechanisms as relevant mediators of this process however Paneni et al. [91] proposed an intriguing molecular pathway that links together chromatin remodeling, ROS production and inflammation of the vessel wall. Over the last few years a number of studies have shown that epigenetic mechanisms, maintained even after glucose normalization, are involved in the transcription of the p65 subunit of NF-kB and on the expression of its target genes: monocyte chemoattractant protein-1 (MCP-1) and vascular cell adhesion molecule-1 (VCAM-1) [93,94]. Moreover, by molecular approaches driving superoxide dismutase-2 (SOD2) overexpression, it has been clearly demonstrated that interfering with ROS generation of mitochondrial origin prevents sugar-induced histone metilation of p65 [95]. Similar results were obtained by using a quite selective mitochondrial antioxidant able to interfere with the recruitment of the Set7 histone methyltransferase to the "chromatinized p65 template" [91]. Moreover, the recent findings that the expression of the mitochondrial adaptor protein $\mathrm{p} 66^{\text {shc }}$ is increased in response to 
hyperglycemia [96,97], has spurred a deep investigation on the involvement of $\mathrm{p} 66^{\text {shc }}$ in ROS-induced "metabolic memory".

Indeed, it has been shown that high glucose challenge leads to persistent $\mathrm{p} 66^{\text {shc }}$ activation and ROS production in vascular cells, despite the return to norm glycaemia [96]. The authors provided clear cut evidences that this event is mediated by epigenetic mechanisms involving promoter demethylation and acetylation of histone 3 [96]. In keeping with the possibility that, despite glucose normalization, vascular damaging signals could be self-maintained by epigenetics, recent studies have also discovered a tight connection among p66shc, the class III histone deacetylase SIRT1 and the tumor transcription factor p53 $[96,98,99]$. Indeed, SIRT1 overexpression in ECs inhibits high glucose-mediated p66shc up-regulation, improves endothelial function and reduces oxidative stress markers [100].

In keeping with the crucial role of p66 ${ }^{\text {shc }}$, p53 and SIRT 1 cross-talk in this context are the following observations: i. p66 $66^{\text {shc }}$ is under the transcriptional control of p53 [101]; ii. SIRT1 inhibition leads to p53 acetylation and to its increased transcription activity [102,103]. To close the circle, Liu et al. [104] demonstrated that Set7, along with Set9 histone methyltransferase, negatively regulate p53 via SIRT1. That epigenetic mechanisms involving SIRT1 are not endothelial specific is supported by the recent finding that SIRT1 over-expression leads to podocyte Claudin-1 "re-writing" resulting in the improvement of renal function in $\mathrm{db} / \mathrm{db}$ mice [105]. Collectively these observations indicate that, despite glucose normalization, epigenetic mechanisms could drive a persistent activation of the intracellular signalling pathways that ultimately lead to endothelial dysfunction and apoptosis [91]. Thus, as an early good glycaemic control could be maintained despite the return to worse metabolic control [84-86], to identify the onset of post-transcriptional modification of histones that change gene expression pattern in diabetic patients should be a future challenge.

\section{Novel options for Diabetes-Associated-Complications}

\section{Adipose tissue-derived stem cells (ASCs)}

The negligible effects of current therapeutic strategies on both endothelial dysfunction and disease progression have highlighted the need for novel therapeutic approaches. Recently, the encouraging results of cell-based therapy using diverse "stem/progenitor" cell populations in preclinical models of vascular diseases have spurred clinicians to exploit these cells in regenerative medicine [6]. For many years bone marrow-derived MSCs (BM-MSCs) have been considered the most relevant sources of stem cells [106,107]. However, the low number of cells recovered and the invasive procedures to obtain them have led to several concerns on the feasibility of exploiting these cells in humans. Different tissue sources have been used, but because of the effortless access by minimally invasive procedures adipose subcutaneous tissue, is nowadays considered a promising alternative source of adipose-derived stem cells (ASCs) for regenerative medicine. This is particularly true for cardiovascular diseases as they can concomitantly stimulate neovascularization, cytoprotection and tissue regeneration [6].

Originally engraftment and terminal differentiation of stem/ progenitor cells were the most often mechanisms studied [106,107]. However, up to now, there is no definitive evidence on their clinical efficacy. In fact up-and-coming data indicate that their therapeutic effectiveness mainly rely on their paracrine effects $[108,109]$. At this regard, Rehman et al. [110] have shown that ASC administration enhances endothelial cell survival in hypoxic conditions and improves ischemic limb perfusion by means of the vascular endothelial growth factor (VEGF) release [110]. Likewise, Nakagami et al. [111] reported that implantation of ASCs into the ischemic hindlimb improves both angiogenic score and capillary density.

As stated above the majority of preclinical studies were performed by using ASCs derived from subcutaneous adipose tissue. However, based on the notion that visceral and subcutaneous adipose tissue possesses distinctive cell autonomous assets [112] it cannot be barred that functional differences in ASCs derived from different fat depots might also exist. Moreover, whether environmental cues, as hyperglycaemia or pathological conditions connoted drastic changes in visceral adipose mass can change their self-renewal capability or secretome is still debated. To address this issue we recently investigated whether visceral adipose tissue-derived ASCs could represent a valuable source of stem cells for regenerative purpose in diabetic condition. To this end ASCs recovered from diabetic patients or cultured in high glucose concentration were assayed for their selfrenewal capability and cytokine production [113]. Stem cell pluripotency are under the control of a network of transcription factors [114], including octamer-binding transcription factor 4 (Oct4) and Nanog [115]. We demonstrated that the diabetic milieu promotes stem cell self-renewal potential by activating NOX and "nontoxic" levels of ROS [113]. Consistent with the crucial role of Oct4 and Nanog in stem cell self-renewing capabilities we demonstrated that ASCs derived from diabetic patients or cultured in the presence of HG express high level of Oct4 and Nanog [113]. By knocking-down NOX we also demonstrated that this pathway is relevant for HG-mediated ASC stemness, measured as "spheroid" formation. Moreover consistently with the ability of ASCs to secrete soluble mediators [116], the de-differentiation status induced by HG resulted in an increase of cytokine production. This effect was partially lost when dedifferentiated ASCs were put in culture in the presence of low glucose concentrations. This implies that hyperglycemic condition can impact ASC stemness and secretion profile. Moreover, the results of this study suggest that omentectomy, along with ameliorating the metabolic profile [117] can offer an additional benefit: the recovered ASCs could be exploited to repair deteriorating tissues. Finally, the results of this study indicate that HG pre-conditioning could be exploited to ex-vivo expand autologous ASCs.

\section{A naturally occurring hormone, the unacylated ghrelin (UnAG) to target ROS-mediated damage}

As stated above vascular remodelling relies on resident ECs and on circulating EPCs $[6,36]$. Thus, changes in EPC number and functional activities, as occurs in diabetes, impact on their delivery to sites of ischemia where new vessel formation might be crucial [36]. This implies that molecules able to revert EPC dysfunction might represent an alternative therapeutic strategy for diabetes-associated vascular complications. Recently it has been shown that gastric-released hormones, such as ghrelin, impact on glucose metabolism as well as on diabetes-associated vascular complications [118]. Indeed, circulating total ghrelin levels are negatively associated with body mass index [119] and obese [120] and in type 2 diabetic patients have a reduced ghrelin secretion [121]. Ghrelin is a 28 amino-acid peptide circulating in two different forms: the acylated (AG) and the unacylated (UnAG) ghrelin [122]. Of particular interest, it has been reported that in clinical settings associated with insulin resistance a relative excess of AG compared to UnAG is common [123]. We have shown that UnAG 
Page 6 of 9

and its cyclic analogue, AZP531 protect vascular cells from ROSmediated damage in diabetic condition [124,125]. In particular we showed that UnAG, but not AG, systemic administration protects diabetic EPCs from oxidative stress and senescence and improves their vasculogenic potential [124]. Moreover, we provided evidences that only UnAG was able to rescue defective EPC mobilization in diabetic patients by restoring eNos activity [124]. Consistently with the antioxidant properties of UnAG, Shimada et al. [126] recently demonstrated that UnAG protects human retinal microvascular endothelial cells from oxidative stress-induced apoptosis through the SIRT-1 signalling pathway. Moreover a prospective study has shown a correlation between circulating des-acyl ghrelin levels and cardiovascular events. In this study Yano et al. [127] demonstrated that a low circulating level of des-acyl ghrelin, is a useful cardiometabolic marker predicting atherosclerosis in elderly hypertensive patients. Based on evidences for the favorable cardiovascular effects of UnAG $[124,125]$ we recently investigated its therapeutic effects on peripheral arterial disease (PAD). PAD is associated with high rate of myocardial infarction, stroke and amputation [128]. The incidence of PAD is expected to increase because of the increasing prevalence of diabetes and population ages [129]. Current surgical approaches are associated with increased perioperative morbidity and mortality [130], while alternative strategies including cell-based therapies or angiogenic growth factor delivery employed so far, were found ineffective [6]. Data obtained in a preclinical model of ischemia demonstrate that UnAG rescues oxidative stress-mediated skeletal muscle damage and induces satellite cell (SC) proliferation and muscle regeneration [131]. We also demonstrated that such effect relies on its ability to induce an efficient antioxidant response mediated by SOD2. By interfering with the expression of SOD2 we also showed that SOD2 strictly controls posttranscriptional mechanisms driven by miR-221/222 to induce skeletal muscle regeneration [131] (Figure 2).

\section{Conclusions and Perspectives}

Defective mitochondrial electron transfer chain along with the increased ROS generation is crucial determinants of cell damage $[132,133]$. This is particularly true in diabetes setting where unbalance of oxidative stress has causal role in its ongoing vascular complications. In the last decade different therapeutic strategies have been exploited to overcome these hurdles, however so far, many of them failed to show clinical benefits $[6,36]$. This implies that the development of a valuable approach is still a thorny challenge. Thus, to design novel therapeutic approaches able to prevent or ameliorate tissue damage, future research efforts should be focused on an in-deep understanding of the mechanisms involved in ROS production and mitochondrial defects. Pre-clinical models of vascular diseases indicate that a naturally occurring hormone, such as UnAG, can act as antioxidant molecules able to reverse vascular damage and to induce skeletal muscle regeneration after ischemia [124,125,131]. In coming years efforts should be directed to evaluate its potential clinical impact in humans. Finally, molecular and pharmacological interventions able to interfere with refractory hyperglycemia resulting in plastic modification of chromatin and the so called "metabolic memory" should be future therapeutic challenges.

\section{Disclosures}

All the authors declare no conflict of interest or duality of interest.

\section{Acknowledgments}

This work was supported by grants obtained by MFB from UnitoCompagnia S. Paolo and from Fondazione perla Ricerca Diabetologica FO. Ri. SID. We thank Dr Patrizia Dentelli for her help with the preparation of figures.

\section{References}

1. Keymel S, Heinen Y, Balzer J, Rassaf T, Kelm M, et al. (2011) Characterization of macro-and microvascular function and structure in patients with type 2 diabetes mellitus. Am J Cardiovasc Dis 1: 68-75.

2. Pries AR, Reglin B, Secomb TW (2005) Remodeling of blood vessels: responses of diameter and wall thickness to hemodynamic and metabolic stimuli. Hypertension 46: 725-731.

3. Martin A, Komada MR, Sane DC (2003) Abnormal angiogenesis in diabetes mellitus. Med Res Rev 23: 117-145.

4. van Rooy M, Pretorius E (2013) Obesity, Hypertension and Hypercholesterolemia as Risk Factors for Atherosclerosis Leading to Ischemic Events. Curr Med Chem.

5. Brizzi MF, Formato L, Dentelli P, Rosso A, Pavan M, et al. (2001) Interleukin-3 stimulates migration and proliferation of vascular smooth muscle cells: a potential role in atherogenesis. Circulation 103: 549-554.

6. Gili M, Orsello A, Gallo S, Brizzi MF (2013) Diabetes-associated macrovascular complications: cell-based therapy a new tool? Endocrine 44: 557-575.

7. Kakehi T, Yabe-Nishimura C (2008) NOX enzymes and diabetic complications. Semin Immunopathol 30: 301-314.

8. Steinberg D, Parthasarathy S, Carew TE, Khoo JC, Witztum JL (1989) Beyond cholesterol. Modifications of low-density lipoprotein that increase its atherogenicity. N Engl J Med 320: 915-924.

9. Krauss RM (2004) Lipids and lipoproteins in patients with type 2 diabetes. Diabetes Care 27: 1496-1504.

10. Shimaoka T, Kume N, Minami M, Hayashida K, Kataoka H, et al. (2000) Molecular cloning of a novel scavenger receptor for oxidized low density lipoprotein, SR-PSOX, on macrophages. J Biol Chem 275: 40663-40666.

11. Horiuchi S, Sakamoto Y, Sakai M (2003) Scavenger receptors for oxidized and glycated proteins. Amino Acids 25: 283-292.

12. Brizzi MF, Dentelli P, Pavan M, Rosso A, Gambino R, et al. (2002) Diabetic LDL inhibits cell-cycle progression via STAT5B and p21(waf). J Clin Invest 109: 111-119.

13. Brizzi MF, Dentelli P, Gambino R, Cabodi S, Cassader M, et al. (2002) STAT5 activation induced by diabetic LDL depends on LDL glycation and occurs via src kinase activity. Diabetes 51: 3311-3317.

14. Howard BV (1994) Lipoprotein metabolism in diabetes. Curr Opin Lipidol 5: 216-220.

15. GugliucciCreriche A, Stahl AJ (1993) Glycation and oxidation of human low density lipoproteins reduces heparin binding and modifies charge. Scand J Clin Lab Invest 53: 125-132.

16. Harja E, Bu DX, Hudson BI, Chang JS, Shen X, et al. (2008) Vascular and inflammatory stresses mediate atherosclerosis via RAGE and its ligands in apoE-/- mice. J Clin Invest 118: 183-194.

17. Ramasamy R, Yan SF, Schmidt AM (2009) RAGE: therapeutic target and biomarker of the inflammatory response--the evidence mounts. J Leukoc Biol 86: 505-512.

18. Wautier MP, Chappey O, Corda S, Stern DM, Schmidt AM, et al. (2001) Activation of NADPH oxidase by AGE links oxidant stress to altered gene expression via RAGE. Am J Physiol Endocrinol Metab 280: E685-694.

19. Yan SF, Ramasamy R, Schmidt AM (2010) The RAGE axis: a fundamental mechanism signaling danger to the vulnerable vasculature. Circ Res 106: 842-853.

20. Matsumura T, Sakai M, Kobori S, Biwa T, Takemura T, et al. (1997) Two intracellular signaling pathways for activation of protein kinase $\mathrm{C}$ are 
involved in oxidized low-density lipoprotein-induced macrophage growth. Arterioscler Thromb Vasc Biol 17: 3013-3020.

21. Li L, Sawamura T, Renier G (2004) Glucose enhances human macrophage LOX-1 expression: role for LOX-1 in glucose-induced macrophage foam cell formation. Circ Res 94: 892-901.

22. Cho HM, Choi SH, Hwang KC, Oh SY, Kim HG, et al. (2005) The $\mathrm{Src} / \mathrm{PLC} / \mathrm{PKC} / \mathrm{MEK} / \mathrm{ERK}$ signaling pathway is involved in aortic smooth muscle cell proliferation induced by glycated LDL. Mol Cells 19: 60-66.

23. Shiu SW, Wong Y, Tan KC (2012) Effect of advanced glycation end products on lectin-like oxidized low density lipoprotein receptor-1 expression in endothelial cells. J Atheroscler Thromb 19: 1083-1092.

24. Sangle GV, Zhao R, Mizuno TM, Shen GX (2010) Involvement of RAGE, NADPH oxidase, and Ras/Raf-1 pathway in glycated LDL-induced expression of heat shock factor-1 and plasminogen activator inhibitor-1 in vascular endothelial cells. Endocrinology 151: 4455-4466.

25. Dumitriu IE, Baruah P, Valentinis B, Voll RE, Herrmann M, et al. (2005) Release of high mobility group box 1 by dendritic cells controls $\mathrm{T}$ cell activation via the receptor for advanced glycation end products. J Immunol 174: 7506-7515.

26. Moser B, Szabolcs MJ, Ankersmit HJ, Lu Y, Qu W, et al. (2007) Blockade of RAGE suppresses alloimmune reactions in vitro and delays allograft rejection in murine heart transplantation. Am J Transplant 7: 293-302.

27. Moser B, Desai DD, Downie MP, Chen Y, Yan SF, et al. (2007) Receptor for advanced glycation end products expression on $\mathrm{T}$ cells contributes to antigen-specific cellular expansion in vivo. J Immunol 179: 8051-8058.

28. Kawahara K, Setoyama K, Kikuchi K, Biswas KK, Kamimura R, et al. (2007) HMGB1 release in co-cultures of porcine endothelial and human T cells. Xenotransplantation 14: 636-641.

29. Lopes-Virella MF, Virella G (2010) Clinical significance of the humoral immune response to modified LDL. Clin Immunol 134: 55-65.

30. Lopes-Virella MF, Hunt KJ, Baker NL, Lachin J, Nathan DM, et al. DCCT/EDIC Study Group (2011) Levels of oxidized LDL and advanced glycation end products-modified LDL in circulating immune complexes are strongly associated with increased levels of carotid intima-media thickness and its progression in type 1 diabetes. Diabetes 60: 582-589.

31. Lopes-Virella MF, Baker NL, Hunt KJ, Lyons TJ, Jenkins AJ, et al. (2012) High concentrations of AGE-LDL and oxidized LDL in circulating immune complexes are associated with progression of retinopathy in type 1 diabetes. Diabetes Care 35: 1333-1340.

32. Bernal-Lopez MR, Garrido-Sanchez L, Gomez-Carrillo V, GallegoPerales JL, Llorente-Cortes V, et al. (2013) Antioxidized LDL antibodies are associated with different metabolic pathways in patients with atherosclerotic plaque and type 2 diabetes. Diabetes Care 36: 1006-1011.

33. Ferretti G, Bacchetti T, Rabini RA, Vignini A, Nanetti L, et al. (2006) Homocysteinylation of low-density lipoproteins (LDL) from subjects with Type 1 diabetes: effect on oxidative damage of human endothelial cells. Diabet Med 23: 808-813.

34. Pirillo A, Uboldi P, Ferri N, Corsini A, Kuhn H, et al. (2012) Upregulation of lectin-like oxidized low density lipoprotein receptor 1 (LOX-1) expression in human endothelial cells by modified high density lipoproteins. Biochem Biophys Res Commun 428: 230-233.

35. Zeoli A, Dentelli P, Rosso A, Togliatto G, Trombetta A, et al. (2008) Interleukin-3 promotes expansion of hemopoietic-derived CD45+ angiogenic cells and their arterial commitment via STAT5 activation. Blood 112: 350-361.

36. Zeoli A, Dentelli P, Brizzi MF (2009) Endothelial progenitor cells and their potential clinical implication in cardiovascular disorders. J Endocrinol Invest 32: 370-382.

37. Fadini GP, Miorin M, Facco M, Bonamico S, Baesso I, et al. (2005) Circulating endothelial progenitor cells are reduced in peripheral vascular complications of type 2 diabetes mellitus. J Am Coll Cardiol 45: 1449-1457.

38. Fadini GP, Sartore S, Albiero M, Baesso I, Murphy E, et al. (2006) Number and function of endothelial progenitor cells as a marker of severity for diabetic vasculopathy. Arterioscler Thromb Vasc Biol 26: 2140-2146.
39. Schmidt-Lucke C, Rossig L, Fichtlscherer S, Vasa M, Britten M, et al. (2005) Reduced number of circulating endothelial progenitor cells predicts future cardiovascular events: proof of concept for the clinical importance of endogenous vascular repair. Circulation 111: 2981-2987.

40. Werner N, Kosiol S, Schiegl T, Ahlers P, Walenta K, et al. (2005) Circulating endothelial progenitor cells and cardiovascular outcomes. N Engl J Med 353: 999-1007.

41. Rosso A, Balsamo A, Gambino R, Dentelli P, Falcioni R, et al. (2006) p53 Mediates the accelerated onset of senescence of endothelial progenitor cells in diabetes. J Biol Chem 281: 4339-4347.

42. Chen Q, Dong L, Wang L, Kang L, Xu B (2009) Advanced glycation end products impair function of late endothelial progenitor cells through effects on protein kinase Akt and cyclooxygenase-2. Biochem Biophys Res Commun 381: 192-197.

43. Liang C, Ren Y, Tan H, He Z, Jiang Q, et al. (2009) Rosiglitazone via upregulation of Akt/eNOS pathways attenuates dysfunction of endothelial progenitor cells, induced by advanced glycation end products. Br J Pharmacol 158: 1865-1873.

44. Sorrentino SA, Bahlmann FH, Besler C, Müller M, Schulz S, et al. (2007) Oxidant stress impairs in vivo reendothelialization capacity of endothelial progenitor cells from patients with type 2 diabetes mellitus: restoration by the peroxisome proliferator-activated receptor-gamma agonist rosiglitazone. Circulation 116: 163-173.

45. Chen J, Huang L, Song M, Yu S, Gao P, et al. (2009) C-reactive protein upregulates receptor for advanced glycationendproducts expression and alters antioxidant defenses in rat endothelial progenitor cells. J Cardiovasc Pharmacol 53: 359-367.

46. Yi LZ, He J, Liang YZ, Yuan DL, Chau FT (2006) Plasma fatty acid metabolic profiling and biomarkers of type 2 diabetes mellitus based on GC/MS and PLS-LDA. FEBS Lett 580: 6837-6845.

47. Stumvoll M, Nurjhan N, Perriello G, Dailey G, Gerich JE (1995) Metabolic effects of metformin in non-insulin-dependent diabetes mellitus. N Engl J Med 333: 550-554.

48. van Bilsen M, van Nieuwenhoven FA (2010) PPARs as therapeutic targets in cardiovascular disease. Expert OpinTher Targets 14: 1029-1045.

49. Trombetta A, Togliatto G, Rosso A, Dentelli P, Olgasi C, et al. (2013) Increase of palmitic acid concentration impairs endothelial progenitor cell and bone marrow-derived progenitor cell bioavailability: role of the STAT5/PPAR gamma transcriptional complex. Diabetes 62: 1245-1257.

50. Urbich C, Kuehbacher A, Dimmeler S (2008) Role of microRNAs in vascular diseases, inflammation, and angiogenesis. Cardiovasc Res 79: 581-588.

51. Havelange V, Garzon R (2010) MicroRNAs: emerging key regulators of hematopoiesis. Am J Hematol 85: 935-942.

52. Fernández-Hernando C, Ramírez CM, Goedeke L, Suárez Y (2013) MicroRNAs in metabolic disease. Arterioscler Thromb Vasc Biol 33: 178-185.

53. Dentelli P, Rosso A, Orso F, Olgasi C, Taverna D, et al. (2010) microRNA-222 controls neovascularization by regulating signal transducer and activator of transcription 5A expression. ArteriosclerThrombVascBiol 30: 1562-1568.

54. Liu X, Cheng Y, Zhang S, Lin Y, Yang J, et al. (2009) A necessary role of miR-221 and miR-222 in vascular smooth muscle cell proliferation and neointimal hyperplasia. Circ Res 104: 476-487.

55. Togliatto G, Trombetta A, Dentelli P, Rosso A, Brizzi MF (2011) MIR221/MIR222-driven post-transcriptional regulation of P27KIP1 and P57KIP2 is crucial for high-glucose- and AGE-mediated vascular cell damage. Diabetologia 54: 1930-1940.

56. Wang XH, Qian RZ, Zhang W, Chen SF, Jin HM, et al. (2009) MicroRNA-320 expression in myocardial microvascular endothelial cells and its relationship with insulin-like growth factor-1 in type 2 diabetic rats. Clin Exp Pharmacol Physiol 36: 181-188.

57. Caporali A, Meloni M, Völlenkle C, Bonci D, Sala-Newby GB, et al. (2011) Deregulation of microRNA-503 contributes to diabetes mellitusinduced impairment of endothelial function and reparative angiogenesis after limb ischemia. Circulation 123: 282-291. 
58. Ziyadeh FN (1993) The extracellular matrix in diabetic nephropathy. Am J Kidney Dis 22: 736-744.

59. Sharma K, Ziyadeh FN (1995) Hyperglycemia and diabetic kidney disease. The case for transforming growth factor-beta as a key mediator. Diabetes 44: 1139-1146.

60. Preisig P (1999) A cell cycle-dependent mechanism of renal tubule epithelial cell hypertrophy. Kidney Int 56: 1193-1198.

61. Herrera RE, Mäkelä TP, Weinberg RA (1996) TGF beta-induced growth inhibition in primary fibroblasts requires the retinoblastoma protein. Mol Biol Cell 7: 1335-1342.

62. Brizzi MF, Dentelli P, Rosso A, Calvi C, Gambino R, et al. (2004) RAGEand TGF-beta receptor-mediated signals converge on STAT5 and p21waf to control cell-cycle progression of mesangial cells: a possible role in the development and progression of diabetic nephropathy. FASEB J 18: 1249-1251.

63. Heydtmann M, Lalor PF, Eksteen JA, Hübscher SG, Briskin M, et al. (2005) CXC chemokine ligand 16 promotes integrin-mediated adhesion of liver-infiltrating lymphocytes to cholangiocytes and hepatocytes within the inflamed human liver. J Immunol 174: 1055-1062.

64. Hofnagel O, Luechtenborg B, Plenz G, Robenek H (2002) Expression of the novel scavenger receptor SR-PSOX in cultured aortic smooth muscle cells and umbilical endothelial cells. Arterioscler Thromb Vasc Biol 22: 710-711.

65. Minami M, Kume N, Shimaoka T, Kataoka H, Hayashida K, et al. (2001) Expression of SR-PSOX, a novel cell-surface scavenger receptor for phosphatidylserine and oxidized LDL in human atherosclerotic lesions. Arterioscler Thromb Vasc Biol 21: 1796-1800.

66. Abel S, Hundhausen C, Mentlein R, Schulte A, Berkhout TA, et al. (2004) The transmembrane CXC-chemokine ligand 16 is induced by IFNgamma and TNF-alpha and shed by the activity of the disintegrin-like metalloproteinase ADAM10. J Immunol 172: 6362-6372.

67. Ludwig A, Hundhausen C, Lambert MH, Broadway N, Andrews RC, et al. (2005) Metalloproteinase inhibitors for the disintegrin-like metalloproteinases ADAM10 and ADAM17 that differentially block constitutive and phorbol ester-inducible shedding of cell surface molecules. Comb Chem High Throughput Screen 8: 161-171.

68. Gutwein P, Abdel-Bakky MS, Schramme A, Doberstein K, Kämpfer-Kolb $\mathrm{N}$, et al. (2009) CXCL16 is expressed in podocytes and acts as a scavenger receptor for oxidized low-density lipoprotein. Am J Pathol 174: 2061-2072.

69. Geissmann F, Cameron TO, Sidobre S, Manlongat N, Kronenberg M, et al. (2005) Intravascular immune surveillance by CXCR6+ NKT cells patrolling liver sinusoids. PLoS Biol 3: el13.

70. Jiang X, Shimaoka T, Kojo S, Harada M, Watarai H, et al. (2005) Cutting edge: critical role of CXCL16/CXCR6 in NKT cell trafficking in allograft tolerance. J Immunol 175: 2051-2055.

71. van der Voort R, van Lieshout AW, Toonen LW, Slöetjes AW, van den Berg WB, et al. (2005) Elevated CXCL16 expression by synovial macrophages recruits memory $\mathrm{T}$ cells into rheumatoid joints. Arthritis Rheum 52: 1381-1391.

72. Jansson AM, Hartford M, Omland T, Karlsson T, Lindmarker P, et al. (2012) Multimarker risk assessment including osteoprotegerin and CXCL16 in acute coronary syndromes. Arterioscler Thromb Vasc Biol 32: 3041-3049.

73. Zhao L, Wu F2, Jin L3, Lu T3, Yang L1, et al. (2014) Serum CXCL16 as a Novel Marker of Renal Injury in Type 2 Diabetes Mellitus. PLoS One 9: e87786.

74. Forbes JM1, Cooper ME (2013) Mechanisms of diabetic complications. Physiol Rev 93: 137-188.

75. UK Prospective Diabetes Study Group (1998) Tight blood pressure control and risk of macrovascular and microvascular complications in type 2 diabetes: UKPDS 38. BMJ 317: 703-713.

76. Gagliardini E, Benigni A (2006) Role of anti-TGF-beta antibodies in the treatment of renal injury. Cytokine Growth Factor Rev 17: 89-96.
77. Wang X, Shaw S, Amiri F, Eaton DC, Marrero MB (2002) Inhibition of the Jak/STAT signaling pathway prevents the high glucose-induced increase in tgf-beta and fibronectin synthesis in mesangial cells. Diabetes 51: 3505-3509.

78. Wanner C, Greiber S, Krämer-Guth A, Heinloth A, Galle J (1997) Lipids and progression of renal disease: role of modified low density lipoprotein and lipoprotein(a). Kidney Int Suppl 63: S102-106.

79. Dentelli P, Rosso A, Zeoli A, Gambino R, Pegoraro L, et al. (2007) Oxidative stress-mediated mesangial cell proliferation requires RAC-1/ reactive oxygen species production and beta4 integrin expression. J Biol Chem 282: 26101-26110

80. Defilippi P, Rosso A, Dentelli P, Calvi C, Garbarino G, et al. (2005) \{beta\}1 Integrin and IL-3R coordinately regulate STAT5 activation and anchorage-dependent proliferation. J Cell Biol 168: 1099-1108.

81. Brizzi MF, Tarone G, Defilippi P (2012) Extracellular matrix, integrins, and growth factors as tailors of the stem cell niche. Curr Opin Cell Biol 24: 645-651.

82. Soldi R, Primo L, Brizzi MF, Sanavio F, Aglietta M, et al. (1997) Activation of JAK2 in human vascular endothelial cells by granulocytemacrophage colony-stimulating factor. Blood 89: 863-872.

83. Kim YS, Jung DH, Sohn E, Kim J, Kim JS (2012) Glycoxidised LDL induced the upregulation of Axl receptor tyrosine kinase and its ligand in mouse mesangial cells. PLoS One 7: e50297.

84. [No authors listed] (1993) The effect of intensive treatment of diabetes on the development and progression of long-term complications in insulindependent diabetes mellitus. The Diabetes Control and Complications Trial Research Group. N Engl J Med 329: 977-986.

85. Writing Team for the Diabetes Control and Complications Trial/ Epidemiology of Diabetes Interventions and Complications Research Group (2003) Sustained effect of intensive treatment of type 1 diabetes mellitus on development and progression of diabetic nephropathy: the Epidemiology of Diabetes Interventions and Complications (EDIC) study. JAMA 290: 2159-2167.

86. Holman RR, Paul SK, Bethel MA, Matthews DR, Neil HA (2008) 10-year follow-up of intensive glucose control in type 2 diabetes. $\mathrm{N}$ Engl J Med 359: 1577-1589.

87. Ceriello A (2009) Hypothesis: the "metabolic memory", the new challenge of diabetes. Diabetes Res Clin Pract 86 Suppl 1: S2-6.

88. El-Osta A (2012) Glycemic memory. Curr Opin Lipidol 23: 24-29.

89. Cooper ME, El-Osta A (2010) Epigenetics: mechanisms and implications for diabetic complications. Circ Res 107: 1403-1413.

90. Reddy MA, Natarajan R (2011) Epigenetic mechanisms in diabetic vascular complications. Cardiovasc Res 90: 421-429.

91. Paneni F, Volpe M, Lüscher TF, Cosentino F (2013) SIRT1, p66(Shc), and Set7/9 in vascular hyperglycemic memory: bringing all the strands together. Diabetes 62: 1800-1807.

92. Pirola L, Balcerczyk A, Tothill RW, Haviv I, Kaspi A, et al. (2011) Genome-wide analysis distinguishes hyperglycemia regulated epigenetic signatures of primary vascular cells. Genome Res 21: 1601-1615.

93. El-Osta A, Brasacchio D, Yao D, Pocai A, Jones PL, et al. (2008) Transient high glucose causes persistent epigenetic changes and altered gene expression during subsequent normoglycemia. J Exp Med 205: 2409-2417.

94. Brasacchio D, Okabe J, Tikellis C, Balcerczyk A, George P, et al. (2009) Hyperglycemia induces a dynamic cooperativity of histone methylase and demethylase enzymes associated with gene-activating epigenetic marks that coexist on the lysine tail. Diabetes 58: 1229-1236.

95. Yoshida N, Okumura K, Aso Y (2005) High serum pentosidine concentrations are associated with increased arterial stiffness and thickness in patients with type 2 diabetes. Metabolism 54: 345-350.

96. Paneni F, Mocharla P, Akhmedov A, Costantino S, Osto E, et al. (2012) Gene silencing of the mitochondrial adaptor p66(Shc) suppresses vascular hyperglycemic memory in diabetes. Circ Res 111:278-289.

97. Pagnin E, Fadini G, de Toni R, Tiengo A, Calò L, et al. (2005) Diabetes induces p66shc gene expression in human peripheral blood mononuclear 
cells: relationship to oxidative stress. J Clin Endocrinol Metab 90 $1130-1136$.

98. Zheng Z, Chen H, Li J, Li T, Zheng B, et al. (2012) Sirtuin 1-mediated cellular metabolic memory of high glucose via the LKB1/AMPK/ROS pathway and therapeutic effects of metformin. Diabetes 61:217-228.

99. Woods A, Dickerson K, Heath R, Hong SP, Momcilovic M, et al. (2005) $\mathrm{Ca} 2+/$ calmodulin-dependent protein kinase kinase-beta acts upstream of AMP-activated protein kinase in mammalian cells. Cell Metab 2: 21-33.

100. Zhou S, Chen HZ, Wan YZ, Zhang QJ, Wei YS, et al. (2011) Repression of P66Shc expression by SIRT1 contributes to the prevention of hyperglycemia-induced endothelial dysfunction. Circ Res 109: 639-648.

101. Kim CS, Jung SB, Naqvi A, Hoffman TA, DeRicco J, et al. (2008) p53 impairs endothelium-dependent vasomotor function through transcriptional upregulation of p66shc. Circ Res 103: 1441-1450.

102. Li L1, Wang L, Li L, Wang Z, Ho Y, et al. (2012) Activation of p53 by SIRT1 inhibition enhances elimination of CML leukemia stem cells in combination with imatinib. Cancer Cell 21: 266-281.

103. Orimo M, Minamino T, Miyauchi H, Tateno K, Okada S, et al. (2009) Protective role of SIRT1 in diabetic vascular dysfunction. Arterioscler Thromb Vasc Biol 29: 889-894.

104. Liu X, Wang D, Zhao Y, Tu B, Zheng Z, et al. (2011) Methyltransferase Set7/9 regulates p53 activity by interacting with Sirtuin 1 (SIRT1). Proc Natl Acad Sci U S A 108: 1925-1930.

105. Hasegawa K, Wakino S, Simic P, Sakamaki Y, Minakuchi H, et al. (2013) Renal tubular Sirt1 attenuates diabetic albuminuria by epigenetically suppressing Claudin-1 overexpression in podocytes. Nat Med 19: 1496-1504.

106. Tateishi-Yuyama E, Matsubara H, Murohara T, Ikeda U, Shintani S, et al. (2002) Therapeutic angiogenesis for patients with limb ischaemia by autologous transplantation of bone-marrow cells: a pilot study and a randomised controlled trial. Lancet 360: 427-435.

107. Gnecchi M, Danieli P, Cervio E (2012) Mesenchymal stem cell therapy for heart disease. Vascul Pharmacol 57: 48-55.

108. Song SY, Chung HM, Sung JH (2010) The pivotal role of VEGF in adipose-derived-stem-cell-mediated regeneration. Expert Opin Biol Ther 10: 1529-1537.

109. Strioga M, Viswanathan S, Darinskas A, Slaby O, Michalek J (2012) Same or not the same? Comparison of adipose tissue-derived versus bone marrow-derived mesenchymal stem and stromal cells. Stem Cells Dev 21 2724-2752.

110. Rehman J, Traktuev D, Li J, Merfeld-Clauss S, Temm-Grove CJ, et al. (2004) Secretion of angiogenic and antiapoptotic factors by human adipose stromal cells. Circulation 109: 1292-1298.

111. Nakagami H, Maeda K, Morishita R, Iguchi S, Nishikawa T, et al. (2005) Novel autologous cell therapy in ischemic limb disease through growth factor secretion by cultured adipose tissue-derived stromal cells. Arterioscler Thromb Vasc Biol 25: 2542-2547.

112. Tran TT, Kahn CR (2010) Transplantation of adipose tissue and stem cells: role in metabolism and disease. Nat Rev Endocrinol 6: 195-213.

113. Dentelli P, Barale C, Togliatto G, Trombetta A, Olgasi C, et al. (2013) A diabetic milieu promotes OCT4 and NANOG production in human visceral-derived adipose stem cells. Diabetologia 56: 173-184

114. Ng HH, Surani MA (2011) The transcriptional and signalling networks of pluripotency. Nat Cell Biol 13: 490-496.

115. Loh YH, Wu Q, Chew JL, Vega VB, Zhang W, et al. (2006) The Oct4 and Nanog transcription network regulates pluripotency in mouse embryonic stem cells. Nat Genet 38: 431-440.
116. Mosna F, Sensebé L, Krampera M (2010) Human bone marrow and adipose tissue mesenchymal stem cells: a user's guide. Stem Cells Dev 19: 1449-1470.

117. Gabriely I, Ma XH, Yang XM, Atzmon G, Rajala MW, et al. (2002) Removal of visceral fat prevents insulin resistance and glucose intolerance of aging: an adipokine-mediated process? Diabetes 51: 2951-2958.

118. Nakazato M, Murakami N, Date Y, Kojima M, Matsuo H, et al. (2001) A role for ghrelin in the central regulation of feeding. Nature 409: 194-198.

119. Cummings DE (2006) Ghrelin and the short- and long-term regulation of appetite and body weight. Physiol Behav 89: 71-84.

120. Tschöp M, Weyer C, Tataranni PA, Devanarayan V, Ravussin E, et al. (2001) Circulating ghrelin levels are decreased in human obesity. Diabetes 50: 707-709.

121. Pöykkö SM, Kellokoski E, Hörkkö S, Kauma H, Kesäniemi YA, et al. (2003) Low plasma ghrelin is associated with insulin resistance, hypertension, and the prevalence of type 2 diabetes. Diabetes 52: 2546-2553.

122. Kojima M1, Hosoda H, Date Y, Nakazato M, Matsuo H, et al. (1999) Ghrelin is a growth-hormone-releasing acylated peptide from stomach. Nature 402: 656-660.

123. Barazzoni R, Zanetti M, Ferreira C, Vinci P, Pirulli A, et al. (2007) Relationships between desacylated and acylated ghrelin and insulin sensitivity in the metabolic syndrome. J Clin Endocrinol Metab 92: 3935-3940.

124. Togliatto G, Trombetta A, Dentelli P, Baragli A, Rosso A, et al. (2010) Unacylated ghrelin rescues endothelial progenitor cell function in individuals with type 2 diabetes. Diabetes 59: 1016-1025.

125. Granata R, Settanni F, Julien M, Nano R, Togliatto G, et al. (2012) Desacyl ghrelin fragments and analogues promote survival of pancreatic $\hat{I}^{2}$ cells and human pancreatic islets and prevent diabetes in streptozotocintreated rats. J Med Chem 55: 2585-2596.

126. Shimada T, Furuta H2, Doi A1, Ariyasu H1, Kawashima H1, et al. (2013) Des-acyl ghrelin protects microvascular endothelial cells from oxidative stress-induced apoptosis through sirtuin 1 signaling pathway. Metabolism.

127. Yano $\mathrm{Y}$, Toshinai K, Inokuchi T, Kangawa K, Shimada K, et al. (2009) Plasma des-acyl ghrelin, but not plasma HMW adiponectin, is a useful cardiometabolic marker for predicting atherosclerosis in elderly hypertensive patients. Atherosclerosis 204: 590-594.

128. American Diabetes Association (2003) Peripheral arterial disease in people with diabetes. Diabetes Care 26: 3333-3341.

129. Jude EB, Eleftheriadou I, Tentolouris N (2010) Peripheral arterial disease in diabetes--a review. Diabet Med 27: 4-14.

130. Hennion DR, Siano KA (2013) Diagnosis and treatment of peripheral arterial disease. Am Fam Physician 88: 306-310.

131. Togliatto G, Trombetta A, Dentelli P, Cotogni P, Rosso A, et al. (2013) Unacylated ghrelin promotes skeletal muscle regeneration following hindlimb ischemia via SOD-2-mediated miR-221/222 expression. J Am Heart Assoc 2: e000376.

132. Finkel $T$ (2005) Radical medicine: treating ageing to cure disease. Nat Rev Mol Cell Biol 6: 971-976.

133. Zaccagnini G, Martelli F, Magenta A, Cencioni C, Fasanaro P, et al. (2007) p66(ShcA) and oxidative stress modulate myogenic differentiation and skeletal muscle regeneration after hind limb ischemia. J Biol Chem 282: 31453-31459.
This article was originally published in a special issue, entitled: "Molecular and Cellular Aspects in Obesity and Diabetes", Edited by Dr. Masayoshi Yamaguchi, Emory University School of Medicine, USA 